Robert W. Vallin, 229K Vincent Science Hall, Slippery Rock University of PA, Slippery Rock, PA 16057, e-mail: rwv@@sruvm.sru.edu *

\title{
MORE ON THE METRIC SPACE OF METRICS
}

\begin{abstract}
In this note we look at a subset of the metric space of metrics for an arbitrary set $X$ and show that in terms of cardinality this can be very large while being extremely small in the porosity sense.
\end{abstract}

\section{Introduction}

We begin with some definitions.

Let $(X, d)$ be a metric space. For $x \in X$ we denote by $B(x, \varepsilon)$ the open ball about $x$ of radius $r$; that is

$$
B(x, \varepsilon)=\{y \in X \mid d(x, y)<\varepsilon\} .
$$

Now let $x \in A \subseteq X$, and fix $R>0$, a real number. We denote by $\gamma(x, R, A)$ the supremum of all real numbers $r>0$ for which there exists a $z \in X$ such that $B(z, r) \subseteq B(x, R)$ and $B(z, r) \cap A=\emptyset$. The porosity of $A$ at $x$ is then given by

$$
p(A, x)=\limsup _{R \rightarrow 0^{+}} \frac{2 \gamma(x, R, A)}{R} .
$$

Porosity values range between zero (not porous) and one (strongly porous). The set $A$ is porous at $x$ if $p(A, x)>0$. A set is called porous if it is porous at each of its points. In a general metric space the class of porous sets is a

\footnotetext{
Key Words: metric space of metrics, very strongly porous

Mathematical Reviews subject classification: Primary: 54E35

Received by the editors March 291995

* The author wishes to thank Chris Freiling for our conversations on this paper and the first referee without whom Theorem 2.1 would not have come about.
} 
subset of the nowhere dense sets. They are not equal; there exists nowhere dense sets which are not porous (see [4]).

If the "lim sup" in the definition of porous is replaced by a "liminf" we have the definition of a set being very porous at a point, very strongly porous sets follow similarly. For a more detailed essay on porosities see [5].

For $X$ a nonempty set we denote by $\operatorname{card}(X)$ the cardinality of $X$ and write $\aleph_{0}$ for the cardinality of the natural numbers. Define $\mathcal{M}=\mathcal{M}(X)$ to be the set of all metrics on $X$. In [2] T. Salát, J. Tóth, and L. Zsilinszky placed a metric on $\mathcal{M}$ defined by

$$
d^{*}\left(d, d^{\prime}\right)=\min \left\{1, \sup _{x, y \in X}\left(d(x, y)-d^{\prime}(x, y)\right)\right\} .
$$

In [2] subspaces $\mathcal{H}_{\alpha}$ and $\mathcal{H}$ were defined by $\mathcal{H}_{\alpha}=\{d: x \neq y \Rightarrow d(x, y) \geq \alpha\}$ and $\mathcal{H}=\bigcup_{\alpha>0} \mathcal{H}_{\alpha}=\bigcup_{k \in \mathbb{N}} \mathcal{H}_{1 / k}$.

Results shown in [2] include $\mathcal{M}$ is a non-complete Baire space and $\mathcal{M} \backslash \mathcal{H}$ is nowhere dense in $\mathcal{M}$. Other results on the metric space of metrics may be found in [1] and [3].

\section{Results}

To gain our results we work with the mostly unexplored set $\mathcal{M} \backslash \mathcal{H}$ and a simple, but seemingly overlooked question. Can we tell, from the set $X$, whether or not $\mathcal{M} \backslash \mathcal{H}$ is nonempty and, if nonempty, how big is it? Two answers are given in the following theorems.

Theorem 1 We have

$$
\operatorname{card}(\mathcal{M} \backslash \mathcal{H})= \begin{cases}0 & \text { if } \operatorname{card}(X)<\aleph_{0} \\ 2^{\operatorname{card}(X)} & \text { if } \operatorname{card}(X) \geq \aleph_{0}\end{cases}
$$

Proof. If $\operatorname{card}(X)=n$, then for each $d \in \mathcal{M}$ there is a natural number $k$ such that $\min _{x \neq y}\{d(x, y)\}>1 / k$. Thus each metric is in $\mathcal{H}$ and $\operatorname{card}(\mathcal{M} \backslash \mathcal{H})=0$.

If $\operatorname{card}(X) \geq \aleph_{0}$, let $A=\left\{a_{n}\right\}$ be a countable, infinite subset of $X$ with infinite complement and let $B$ be a subset of $X \backslash A$ with $\operatorname{card}(B)=\operatorname{card}(X)$. Place the following metric on $X$ :

$$
\rho_{B}(x, y)=\left\{\begin{array}{cl}
0 & x=y \\
1 & x \text { or } y \text { is in } X \backslash A, x, y \notin B, x \neq y \\
2 & x \text { or } y \text { is in } B, x \neq y \\
\max \{1 / n, 1 / m\} & x=a_{n}, y=a_{m} .
\end{array}\right.
$$


It is easy to verify that $\rho_{B}$ is a metric and that $\rho_{B} \neq \rho_{B^{\prime}}$ if $B \neq B^{\prime}$. Now, pick $\varepsilon>0$. For this $\varepsilon$ there is an $N=N(\varepsilon)$ such that for all $n \geq N, 1 / n<\varepsilon$. Thus for $n, m \geq N, \rho_{B}\left(a_{n}, a_{m}\right)<\varepsilon$. This says there are points in $X$ arbitrarily close to one another so $\rho_{B} \notin \mathcal{H}$. There are $2^{\operatorname{card}(X)}$ many choices for $B$ so we can see

$$
2^{\operatorname{card}(X)} \leq \operatorname{card}(\mathcal{M} \backslash \mathcal{H}) \leq \operatorname{card}(\mathcal{M}) \leq 2^{\operatorname{card}(X)}
$$

We now contrast this "largeness" of $\mathcal{M} \backslash \mathcal{H}$ in cardinality terms by showing that $\mathcal{M} \backslash \mathcal{H}$ is in fact as very porous as possible.

Theorem 2 The set of metrics on the space $X$ with values arbitrarily close to zero is a very strongly porous subset in the space of all metrics on $X$.

ProOF. We only need concern ourselves with the case that $\operatorname{card}(X) \geq \aleph_{0}$ so assume this is so. Let $\rho$ be an arbitrary metric in $\mathcal{M} \backslash \mathcal{H}$ and let $\varepsilon_{n}$ be a sequence of positive values which converge to zero as $n$ approaches infinity. Fix $n$. Let $\rho_{n}$ be the metric defined by

$$
\rho_{n}(x, y)=\left\{\begin{array}{cl}
0 & \text { if } x=y \\
\rho(x, y)+\frac{\varepsilon_{n}}{2} & \text { if } x \neq y
\end{array}\right.
$$

Note two things: First that $\rho_{n} \in \mathcal{H}$ and second that $d^{*}\left(\rho, \rho_{n}\right)=\frac{1}{2} \varepsilon_{n}$, so that $\rho_{n} \in B\left(\rho, \varepsilon_{n}\right)$. It is easy to see that since $\rho_{n} \in \mathcal{H}_{\varepsilon_{n} / 2}$, we have

$$
B\left(\rho_{n},\left(\varepsilon_{n} / 2\right)\right) \subset \mathcal{H} .
$$

So in terms of porosity

$$
p(\mathcal{M} \backslash \mathcal{H}, \rho) \geq \lim _{n \rightarrow \infty} 2 \frac{\left(\varepsilon_{n} / 2\right)}{\varepsilon_{n}}=1 .
$$

Since $\rho$ and $\left\{\varepsilon_{n}\right\}$ were arbitrary, this actually shows $\mathcal{M} \backslash \mathcal{H}$ is a very strongly porous subset of $\mathcal{M}$.

What we have here is a metric space and a very porous subset which have equal cardinality. The next question to ask is if this is always the case. First note that in $(X, d)$ with $d \in \mathcal{H}$ the only porous set is the empty set. This is the case also for some metrics in $\mathcal{M} \backslash \mathcal{H}$ when $X$ is infinite (see Theorem 2.1).

There are however metric spaces where the cardinality of the nonempty very porous sets is neither zero nor the cardinality of the space, e.g. if

$$
X=\left\{2^{-n}: n \in \mathbb{N}\right\} \cup\{0\}
$$

with the usual topology, then the only very porous nonempty set in $X$ is $\{0\}$. 
Question 1 Can one, for any give space $X$, describe the set of metrics on $X$ such that the cardinality of the very porous sets equals the cardinality of the space?

\section{References}

[1] T. Salát, J. Tóth, and L. Zsilinszky, On cardinality of sets of metrics generating metric spaces of prescribed properties, Annales Univ. Sci. Budapest, 35 (1992), 15-21.

[2] T. Salát, J. Tóth, and L. Zsilinszky, Metric space of metrics defined on a given set, Real Anal. Exch., 18 no. 1 (1992-1993), 225-231.

[3] T. Salát, J. Tóth, and L. Zsilinszky, On the structure of the space of metrics defined on a given set, Real Anal. Exch, 19 no. 1 (1993-1994), $321-327$.

[4] L. Zajíček, Sets of porosity and sets of $\sigma$-porosity (q), Časopis Pěst. Mat., 101 (1976), 350-359.

[5] L. Zajíček, Porosity and $\sigma$-porosity, Real Anal. Exch, 13 no. 2 (19871988), 314-350. 